\title{
Using X-ray Crystallography to Fight Back Against Pneumoviruses
}

\section{Jason S. McLellan The University of Texas at Austin}

Respiratory syncytial virus (RSV) and metapneumovirus (MPV) are the two human pathogens in the recently created Pneumoviridae family of the order Mononegavirales. Both viruses are major causes of severe lower respiratory tract infections, particularly in young children. Our structural studies on the fusion glycoproteins of both viruses have allowed for the development of promising new vaccine candidates and small-molecule fusion inhibitors. Some of these vaccine antigens have also provided insights into humoral immune responses and facilitated the isolation of potent antibodies and nanobodies that are being developed for passive vaccination. This talk will summarize the recent advances in the field that were facilitated by X-ray crystallography. 